Table. Data from NOBILITY at weeks 52 and 76 using several response definitions

\begin{tabular}{|c|c|c|c|c|c|c|}
\hline \multirow[t]{2}{*}{ Definition of response } & \multicolumn{3}{|c|}{ Week 52} & \multicolumn{3}{|c|}{ Week 76} \\
\hline & OBI $(n=63)$ & $\mathrm{PBO}(\mathrm{n}=62)$ & Diff. & OBI $(n=63)$ & PBO $(n=62)$ & Diff. \\
\hline $\begin{array}{l}\text { NOBILITY complete response } \\
\text { UPCR }<0.5, \mathrm{SCr} \leq \mathrm{ULN} \text { and not increased }>15 \% \text { from baseline } \mathrm{SCr} \text {, } \\
\text { and }<10 \mathrm{RBC} / \mathrm{hpf} \text { without casts }\end{array}$ & $35 \%$ & $23 \%$ & $12 \%{ }^{*}$ & $40 \%$ & $18 \%$ & $22 \%$ ** \\
\hline $\begin{array}{l}\text { REGENCY complete response } \\
\text { UPCR }<0.5, \mathrm{SCr} \leq \mathrm{ULN} \text { and not increased }>25 \% \text { from baseline } \mathrm{SCr}\end{array}$ & $43 \%$ & $29 \%$ & $14 \% *$ & $54 \%$ & $31 \%$ & $23 \%$ ** \\
\hline $\begin{array}{l}\text { UPCR }<0.8 \text { only } \\
\text { UPCR }<0.8 \text { with SCr requirement } \\
\text { UPCR }<0.8 \text { and } S C r \leq U L N \text { or not increased }>15 \% \text { from baseline } \mathrm{SCr}\end{array}$ & $\begin{array}{l}64 \% \\
60 \%\end{array}$ & $\begin{array}{l}48 \% \\
48 \%\end{array}$ & $\begin{array}{l}15 \%^{*} \\
12 \%{ }^{*}\end{array}$ & $\begin{array}{l}64 \% \\
64 \%\end{array}$ & $\begin{array}{l}47 \% \\
45 \%\end{array}$ & $\begin{array}{l}17 \% \%^{*} \\
18 \%^{* *}\end{array}$ \\
\hline $\begin{array}{l}\text { NOBILITY overall response } \\
\text { CRR or } \geq 50 \% \text { reduction in UPCR }{ }^{a} \text { with SCr not increased }>15 \% \text { from } \\
\text { baseline and urinary RBCs not increased }>50 \% \text { from baseline }\end{array}$ & $56 \%$ & $36 \%$ & $20 \%$ ** & $51 \%$ & $29 \%$ & $22 \%$ ** \\
\hline $\begin{array}{l}\text { REGENCY overall response } \\
\text { CRR or } \geq 50 \% \text { reduction in UPCR }{ }^{a} \text { with SCr not increased }>25 \% \text { from } \\
\text { baseline }\end{array}$ & $68 \%$ & $45 \%$ & $23 \%$ ** & $67 \%$ & $50 \%$ & $17 \%$ ** \\
\hline
\end{tabular}

OBI, obinutuzumab; PBO, placebo.

${ }^{*} \mathrm{P}<0.2$ vs. placebo group. ${ }^{* *} \mathrm{P}<0.05$ vs. placebo group.

${ }^{a} \geq 50 \%$ reduction in UPCR to a value $<1$ ( $<3$ if the baseline UPCR was $\geq 3$ ). All response definitions required no use of rescue medications or early withdrawal.

\section{OP0167 SUCCESSFUL WITHDRAWAL OF MYCOPHENOLATE MOFETIL IN QUIESCENT SLE: RESULTS FROM A RANDOMIZED TRIAL}

E. Chakravarty ${ }^{1}$, T. Utset ${ }^{2}$, D. L. Kamen ${ }^{3}$, G. Contreras ${ }^{4}$, W. J. Mccune ${ }^{5}$, K. C. Kalunian ${ }^{6}$, C. Aranow ${ }^{7}$, M. Clowse ${ }^{8}$, E. Goldmuntz ${ }^{9}$, J. Springer ${ }^{9}$, L. KeyesElstein $^{10}$, B. Barry ${ }^{10}$, A. Pinckney ${ }^{10}$, J. James ${ }^{1}$ on behalf of ALE06 Working Group. ${ }^{1}$ OMRF, Oklahoma City, United States of America; ${ }^{2} U$. Chicago, Chicago, United States of America; ${ }^{3}$ MUSC, Charleston, United States of America; ${ }^{4}$ U. Miami, Miami, United States of America; ${ }^{5}$ U. Michigan, Ann Arbor, United States of America; ${ }^{6}$ UCSD, La Jolla, United States of America; ${ }^{7}$ Feinstein Institute, Manhasset, United States of America; ${ }^{8}$ Duke, Durham, United States of America; ${ }^{9}$ NIHINIAID, Rockville, United States of America; ${ }^{10}$ Rho, Durham, United States of America

Background: Trials and clinical observations have demonstrated the efficacy of mycophenolate mofetil (MMF) for SLE treatment. Long-term use of MMF is associated with adverse events, pregnancy risks, drug monitoring, and increased cost. Current management continues therapy indefinitely. Whether immunosuppression may be safely withdrawn or whether risks of withdrawal outweigh the benefits of continuation is unknown.

Objectives: To compare rates of clinically significant disease reactivation (CSDR), major flares, and all flares in patients with quiescent SLE on stable MMF randomized to maintain or withdraw MMF. The goal is to provide guidance for clinicians and patients on the risks of MMF withdrawal.

Methods: Adults with quiescent SLE (SELENA-SLEDAI without serologies $<4$ ) receiving MMF for $\geq 2$ years for nephritis or $\geq 1$ year for non-nephritis were randomized 1:1 to unblinded MMF (maintenance arm, MA) or to a 12-week taper off MMF (withdrawal arm, WA) and followed through 60 weeks. Subjects were on stable hydroxychloroquine; steroids limited to $\leq 10 \mathrm{mg}$. CSDR, defined as a SLEDAI flare requiring immunosuppression, BILAG flares and adverse events were assessed. Event rates and time to flare were compared using Kaplan-Meier. Results: 102 subjects were randomized (50 MA, 52 WA); 1 subject in each arm was ineligible and 10 terminated early (7 MA, $3 \mathrm{WA})$. Mean disease duration was 13 years; $76 \%$ had a history of nephritis; mean baseline SLEDAI was 2.2. 5 MA subjects (10\%) had CSDR, compared to 9 WA (17\%). Median time to CDSR

Table 1. Baseline and Demographic Characteristics

\begin{tabular}{lccc}
\hline & Maintenance arm & Withdrawal arm & Total \\
\hline Randomized & 50 & 52 & 102 \\
Female, n (\%) & $39(78)$ & $47(90)$ & $86(84)$ \\
White, n (\%) & $25(50)$ & $19(37)$ & $44(43)$ \\
Black, $\mathrm{n}(\%)$ & $19(38)$ & $22(42)$ & $41(40)$ \\
Hispanic/Latino, n (\%) & $10(20)$ & $12(23)$ & $22(22)$ \\
Age, Years, mean (SD) & $42.4(12.9)$ & $41.6(12.5)$ & $42.0(12.6)$ \\
Disease Duration, Years, mean (SD) & $13.6(8.2)$ & $12.2(7.9)$ & $12.9(8.0)$ \\
H/O Lupus Nephritis, n (\%) & $40(80)$ & $38(73)$ & $78(76.5)$ \\
On Baseline Steroids, n (\%) & $18(36)$ & $23(44)$ & $41(40)$ \\
Prednisone Dose, mg, mean (SD) & $4.8(2.7)$ & $3.3(1.7)$ & $4.0(2.3)$ \\
MMF Duration, Years, mean (SD) & $6.8(4.3)$ & $6.4(4.3)$ & $6.6(4.3)$ \\
Baseline MMF Dose, mg, mean & 1,612 & 1,668 & 1,640 \\
SELENA-SLEDAl*, mean (SD) & $2.4(1.76)$ & $1.9(1.76)$ & $2.2(1.77)$ \\
Positive DsDNA, n (\%) & $35(70)$ & $27(52)$ & $62(61)$ \\
Low C3 $^{1}, \mathrm{n}(\%)$ & $14(28)$ & $9(17)$ & $23(23)$ \\
Low C4 $^{1}$, n (\%) & $6(12)$ & $5(10)$ & $11(11)$ \\
\hline
\end{tabular}

was 38 weeks in both arms. BILAG A flares occurred in 1MA subject (pancreatitis) vs. 4 WA (cranial neuropathy, panniculitis, 2 nephritis). Kaplan-Meier curves overlapped for CDSR, BILAG A flares, and all SLEDAl flares (Figure). Based on these data, we are $86 \%$ confident that the increased risk of CDSR with MMF withdrawal is less than $15 \%$ over 60 weeks. AEs were similar between groups; infections occurred more commonly in MA (63 vs. 49).

Conclusion: In this cohort of subjects with quiescent SLE on long term MMF serious flares occurred infrequently in subjects continuing or withdrawing MMF without differences in time to flare. MMF withdrawal may be considered in subjects with prolonged quiescent disease.

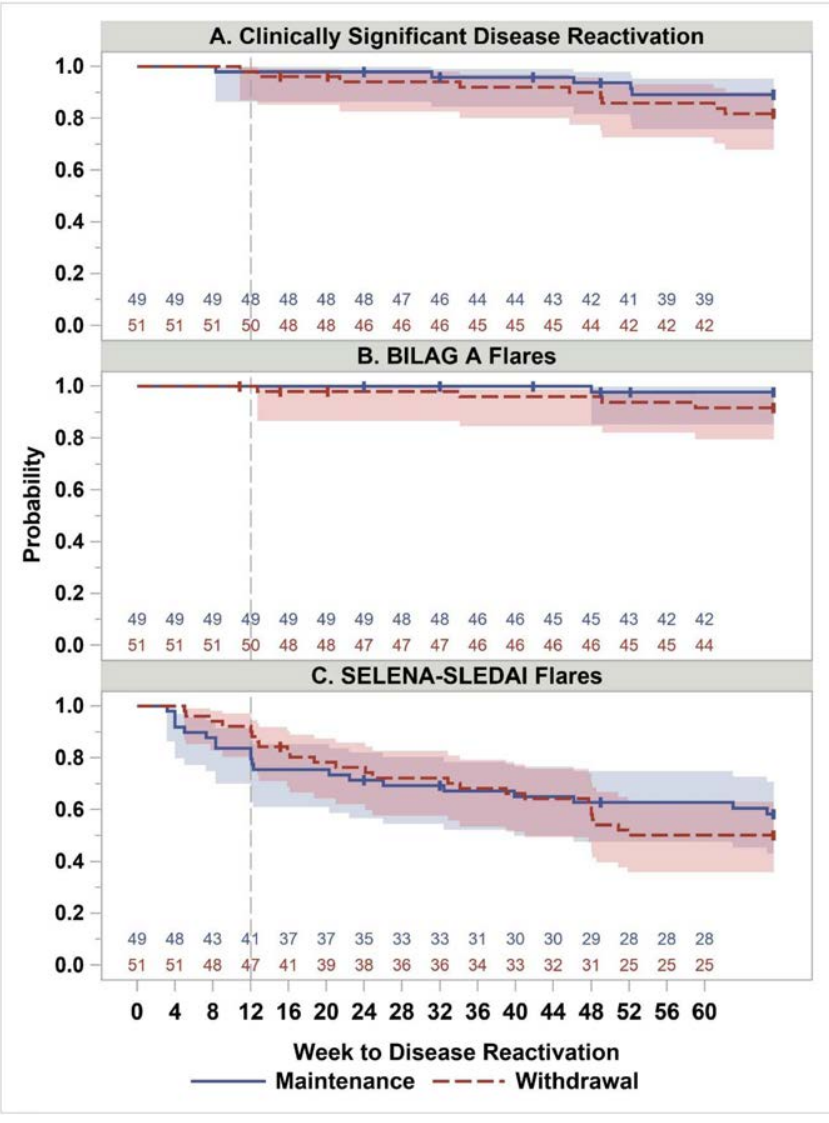

Figure. Kaplan-Meier Estimates of Flare Endpoints

Disclosure of Interests: Eliza Chakravarty: None declared, Tammy Utset: None declared, Diane L Kamen Consultant of: Consulted on SLE survey development for Lilly and consulted on SLE trial protocol development for EMD Serono in 2019, Gabriel Contreras Grant/research support from: Genentech, Merck, Consultant of: Genentech, Merck, William Joseph McCune: None declared, Kenneth 
C Kalunian: None declared, Cynthia Aranow: None declared, Megan Clowse Grant/research support from: GSK, Pfizer, Consultant of: UCB, Astra-Zeneca, Speakers bureau: UCB, Ellen Goldmuntz: None declared, Jessica Springer: None declared, Lynette Keyes-Elstein: None declared, Bill Barry: None declared, Ashley Pinckney: None declared, Judith James: None declared DOI: 10.1136/annrheumdis-2020-eular.5110

\section{Crystals and bones: A selection of best abstracts}

\section{OP0168 \\ CARDIOVASCULAR EVENT ASSOCIATED WITH INITIATING ALLOPURINOL AND FEBUXOSTAT - ACUTE GOUT ATTACK AND CARDIOVASCULAR GOUT ATTACK}

H. J. Kim ${ }^{1}$, H. S. Ahn ${ }^{1}$, J. Kim ${ }^{2}$, B. Ghang ${ }^{2} .{ }^{1}$ College of Medicine, Korea University, Seoul, Korea, Rep. of (South Korea); ${ }^{2}$ Jeju National University School of Medicine, Jeju, Korea, Rep. of (South Korea)

Background: When serum uric acid rapidly increases or decreases due to such as alcohol consumption or fasting, free urate crystals are formed, which induce an acute joint inflammation referred to as an acute gout attack. In $86.4 \%$ of patients with gout, dual energy computed tomography demonstrated the deposition of urate crystals in vasculature, and urate crystals have been observed in coronary arteries and various tissues in $10.9 \%$ of patients with a heart transplant. However, whether hyperuricemia and urate crystal directly cause cardiovascular disease is not well known. We previously reanalyzed the CARES trial to calculate the mortality rates based on the median duration of exposure to study drugs and the median follow-up duration. A sharp increase in mortality was observed after allopurinol and febuxostat were discontinued. Even in view of the Sick-Stopper Effect, for about 40-fold increase in mortality following drug discontinuation, we postulated that the sharp increase in mortality may be associated with rapid changes in uric acid level (rebound hyperuricemia), and that withdrawal of hypouricemic agent leads to an acute inflammatory response due to an abrupt increase in serum uric acid level with subsequent free urate crystal formation in the cardiovascular system (cardiovascular gout attack)(1).

Objectives: Based on this hypothesis that some cardiovascular events may be cardiovascular gout attacks, we investigated the association between the cardiovascular event and initiation of hypouricemic agent, when is accompanied by acute gout attack and/or fluctuation of uric acid, in gout patients.

Methods: Using the Korean National Health Insurance Service (KNHIS) database, which covers the entire Korean population, we conducted a population-based cohort study among gout patients who initiated allopurinol or febuxostat between 2012 and 2018. The initiators were defined as those who had no prior dispensing of any urate-lowering therapy for at least 60 months before the first dispensing date (i.e. index date) of either allopurinol or febuxostat. We excluded patients with a diagnosis of cancer and patients treated with benzbromarone. We investgated a composite cardiovascular event of hospitalized myocardial infarction, ischemic stroke, and cerebral hemorrhage.

Results: We identified hospitalizations for cardiovascular event (acute myocardial infarction ( $n=3538)$, cerebral infarction $(n=5127)$, and cerebral hemorrhage

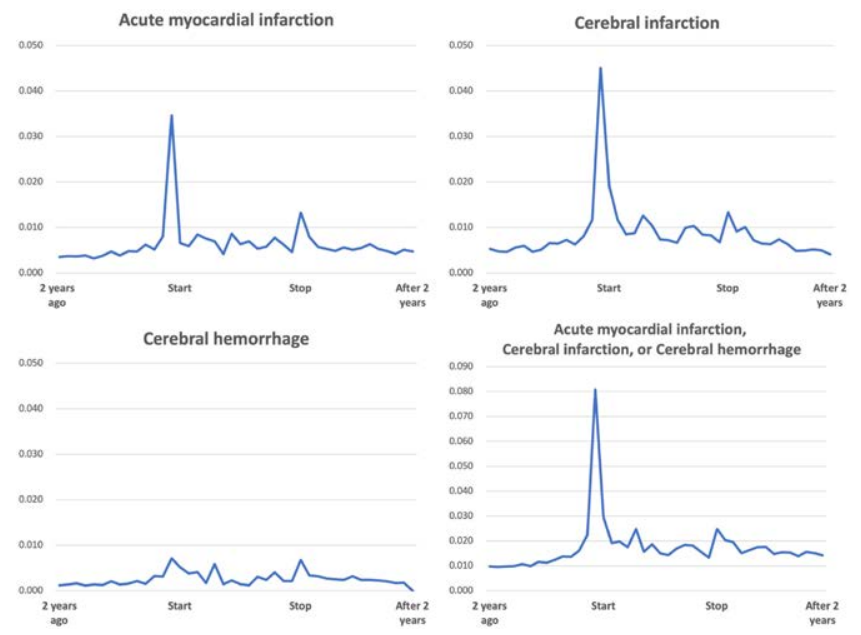

Figure. The cardiovascular event in gout patients who initiated and discontinued allopurinol and febuxostat. $(n=1593))$ that occurred within 2 year before and 2 year after initiation of allopurinol or febuxostat. Of these, 4333 cardiovascular event $(0.014$ per person-time, $95 \%$ confidence interval $[\mathrm{Cl}], 0.014$ to 0.015$)$ occurred in 2 years before initiation of allopurinol or febuxostat, 1032 cardiovascular event $(0.081$ per person-time, $95 \%$ confidence interval [Cl], 0.076 to 0.086 ) occurred in 30 days before initiation of allopurinol or febuxostat. And, 83 cardiovascular event $(0.030$ per person-time $95 \% \mathrm{Cl}, 0.024$ to 0.037 ) occurred in 7 days after initiation of allopurinol or febuxostat. (Figure)

Conclusion: Initiation of febuxostat and allopurinol was significantly associated with acute myocardial infarction, cerebral infarction, and cerebral hemorrhage. Considering that allopurinol and febuxostat usually are initiated a few weeks after a acute gout attack. Acute gout attack and/or fluctuation of uric acid level might be significantly associated with cardiovascular event. Some of cardiovascular events might be cardiovascular gout attack.

\section{References:}

[1] Ghang B, Ahn SM, Kim J, Kim YG, Lee CK, Yoo B. Discontinuing febuxostat might cause more deaths than continuing febuxostat: the untold story from the CARES trial. Rheumatology (Oxford). 2019.

Disclosure of Interests: None declared

DOI: 10.1136/annrheumdis-2020-eular.1909

\section{OP0169 \\ AMPUTATION PROCEDURES IN PATIENTS WITH GOUT COMPARED TO PATIENTS WITH DIABETES}

\section{B. Lamoreaux ${ }^{1}$, M. Francis-Sedlak ${ }^{1}$, R. Holt ${ }^{1}{ }^{1}$ Horizon Therapeutics, Lake} Forest, United States of America

Background: Gout is the most common inflammatory arthritis in the United States (U.S.) and is associated with specific comorbidities, including hypertension, renal disease, cardiovascular disease, hyperlipidemia, and metabolic syndrome (1). This set of comorbidities is known for carrying an increased risk of macrovascular complications (e.g., myocardial infarction, stroke) and peripheral limb problems (e.g., skin ulcers, amputations). Diabetics are known to have an elevated risk of undergoing ulcer and amputation procedures, which have been shown to increase morbidity and mortality in this population $(2,3)$. It is currently not known if patients with gout have an elevated independent risk for limb amputations or whether gout potentiates amputation rates in patients with diabetes.

Objectives: To assess and compare the rate of amputation procedures conducted in patients with gout, diabetes, both gout and diabetes, and neither gout nor diabetes.

Methods: In September 2019, a large U.S. claims database (includes data from 190 million patients over 7 years, TriNetX "Diamond" network) was used to determine amputation rates in patients with gout and diabetes. TriNetX only provides aggregate data and statistical summaries of de-identified patient information. Initial cohorts were developed to understand the amputation rate in patients with gout, regardless of diabetes comorbidity $(n=4,467,721)$, and the amputation rate in patients with diabetes, regardless of gout comorbidity ( $n=$ $25,972,726)$. Subsequently, the following four cohorts were constructed to isolate these two diseases: 1) presence of gout without diabetes $(n=2,471,430)$, 2) presence of diabetes without gout $(n=23,976,435), 3)$ presence of both gout and diabetes ( $n=1,996,291)$, and 4$)$ absence of both gout and diabetes (control cohort, $n=144,705,645$ ). Demographic features of these groups were tabulated and amputation (foot, toes, hand, fingers) rates were calculated using procedural codes reported in each group.

Results: The overall rate of amputations in patients with gout $(0.434 \%)$ was similar to the amputation rate in patients with diabetes $(0.484 \%)$. However when separating these patients into distinct, non-overlapping cohorts, the amputation rate in patients with gout but not diabetes $(0.162 \%)$ differed from the rate in patients with diabetes but not gout $(0.461 \%)$. The control population (no gout or diabetes) had an amputation rate of $0.035 \%$. Unexpectedly, patients with both gout and diabetes had an amputation rate of $0.770 \%$, the highest of all groups examined.

Conclusion: Gout is increasingly being linked to unfavorable cardiovascular, renal, and metabolic complications. Our analysis showed that having gou also increased the likelihood of undergoing an amputation procedure. Patients with gout but not diabetes suffered an approximately 3 -fold increase in amputations compared to patients without either disease. Additionally, patients with both gout and diabetes had a notably increased risk of amputation compared to patients with only diabetes (no gout). Because amputations are an unfavorable outcome associated with procedural complication risk and long-term sequelae this apparent increased risk of amputation in patients with gout warrants further exploration.

References:

[1] Dalbeth N, et al. Nature Reviews Disease Primers, 2019;5(69):1-17

[2] Geiss LS, et al. Diabetes Care, 2019;42:50-54

[3] Moulik PK, et al. Diabetes Care, 2003;26:491-494 